\title{
COMMUNICATION
}

\section{Ostéotomie alvéolaire segmentaire d'impaction postérieure mandibulaire : présentation d'un cas clinique et gestion de l'échec.}

\section{Castro $\mathbf{R}^{1}$, Lan $\mathbf{R}^{1,2}$, Haen $\mathbf{P}^{3}$, Roche-Poggi $\mathbf{P}^{1}$}

1. Service de Chirurgie Orale, pavillon odontologie, Timone - AP-HM - Marseille

2. UMR 7268 ADES, Aix-Marseille/EFS/CNRS - Faculté de médecine-secteur Nord - Marseille

3. Service de Chirurgie Maxillo-faciale, Hôpital d'instruction des armées Laveran - AP-HM - Marseille

\section{Introduction}

l'insuffisance d'espace inter arcades causée par l'absence de restaurations prothétiques réalisées après la perte de dents aboutit généralement à l'égression du secteur alvéolo-dentaire antagoniste et perturbe fortement la courbe d'occlusion et la hauteur occlusale prothétique utilisable (HOPU). Plusieurs méthodes chirurgicales et prothétiques existent pour compenser cette perte d'espace et rétablir une dimension verticale rendant possible la confection de prothèses. Parfois, le recours à des techniques d'ostéotomie segmentaire trouvent leurs indications dans l'arsenal thérapeutique du chirurgien orale. Ce geste chirurgicale localisé au secteur postérieur mandibulaire, décrit en 1978 (1), se limitant à l'étage alvéolaire présente des indications rares, lorsque les moyens orthodontiques deviennent insuffisants pour engendrer les mouvements dentaires désirés et rétablir une fonction occlusale harmonieuse. Cependant, la technique n'est pas sans risques et le secteur postérieur mandibulaire, de part ses particularités anatomiques, est hautement exposé à une possibilité d'échec.

\section{Observation}

ce cas clinique concerne une patiente de 43 ans, sans antécédents médicaux, fumeuse modérée (5 paquet/ année), ayant bénéficié 2 ans auparavant d'une ostéotomie segmentaire d'impaction du secteur 4 comprenant les dents 45, 46, 47, traitées endodontiquement, fortement égréssées et ne permettant aucunes reconstructions prothétiques antagonistes faute d'espace verticale disponible. En situation d'échec, puisque 2 foyers de pseudarthroses persistaient au niveau du trait de section osseux, le même geste a été retenté. En effet, le bloc alvéolo-dentaire, en l'état, mobile et sujet aux infections, n'était pas viable. Une désinfection, un curetage et une reconsolidation du bloc par ostéosynthèse était la meilleur solution de derniers recours. 6 semaines plus tard, tout le bloc osseux alvéolo-dentaire s'est finalement nécrosé et une chirurgie de parage et nettoyage a permis de retrouver une muqueuse assainie malgré la perte de 3 dents et un volume osseux conséquent. Une réhabilitation par prothèse amovible a été réalisé 2 mois après la chirurgie d'assainissement.

\section{Discussion}

la littérature est pauvre en ce qui concerne cette technique d'ostéotomie postéro-latérale mandibulaire. Des techniques semblables comme celles de Schuchardt (2) ou Wassmund ou même l'ostéotomie mandibualire segmentaire alvéolaire antérieure sont plus décrites, présentent des taux de réussites plus élevés et des suites plus prédictibles. Pour ce cas, d'autres méthodes impliquant l'avulsion des dents et une réhabilitation implanto-portée auraient pu être probablement plus bénéfique pour la patiente.

castro.romain1@gmail.com

\footnotetext{
Références

(1) Peterson LJ et al. Posterior mandibular segmental alveolar osteotomy. J Oral Surg. 1978 Jun;36(6):454-8

(2) James D. Kassolis et al. The segmental osteotomy may provide an alternative treatment to the removal of severely malposed implants or their compromised prosthetic restoration. J Periodontol 2003;74:529-536

(2) Baeg et al. Posterior maxillary segmental osteotomy for management of insufficient intermaxillary vertical space and intermolar width discrepancy:
} a case report. Maxillofacial Plastic and Reconstructive Surgery (2016) 38:28

(C) The authors, published by EDP Sciences. This is an Open Access article distributed under the terms of the Creative Commons Attribution License 4.0 (http://creativecommons.org/licenses/by/4.0/). 